\title{
ROLE OF PLATELET RICH PLASMA THERAPY ON ROTATOR CUFF DISEASE
}

\author{
Metwaly M 1, Nassar N.1, Khaled H.1, El Mikkawy D. ${ }^{1}$ \\ and Sakr H. ${ }^{2}$
}

\author{
1 Physical medicine, \\ Rheumatology and \\ Rehabilitation department, Ain \\ Shams University. \\ ${ }^{2}$ Radio-diagnosis departement, \\ Ain Shams University. \\ Cairo, Egypt
}

Corresponding Author:

Mohammed M. Metwaly

Mobile: (+2) 01146163682

E-mail:

m.mousta2011@yahoo.com

Received: $30 / 7 / 2020$

Accepted: $20 / 8 / 2020$

Online ISSN: 2735-

\begin{abstract}
:
Background: There is lack of evidence from randomized clinical trials that had assisted the efficacy of PRP in treating of rotator cuff diseases (RCD).
\end{abstract}

Aim of the work: To study the effect of PRP injection on functional improvement on RCD, and to study the role of proinflammatory and angiogenesis-related cytokines as a pathogenic factor in $R C D$.

Patients and methods:The study was conducted on 40 patients with RCD. All patients subjected to: clinical shoulder examination, clinical scoring including; (VAS and Los Angeles Shoulder score), cytokine assay of vascular endothelial growth factor (VEGF) and interleukin one beta (IL-1 $\beta)$, and diagnostic musculoskeletal ultrasound examination (MSU). All patients underwent two doses of ultrasound guided PRP injection with 4 weeks in-between. Reevaluation after 4 weeks from first injection was done using clinical scoring, cytokine assay and MSU. Third assessment was done 3 months from first injection using clinical scoring and MSU.

Results: There was a statistically high significant decrease in pain, improvement of range of motion (ROM), power and functional state in all participants after 2 doses of PRP injection and rehabilitation program. In addition, there was a highly statistical significant decrease of cytokines serum levels after 4 weeks. There was a highly significant improvement regarding MSU grading. There was positive correlation between the serum levels of cytokines and VAS and negative correlation between the serum levels of cytokines and other clinical scores. There was positive correlation between the serum levels of VEGF and MSU grading.

Conclusion:PRP therapy is an effective treatment for $R C D$ and lead to functional improvement. There is a strong correlation between levels of cytokines (IL-1 $\beta$ and VEGF) and clinical scores before injection and 4 weeks following first injection. There is strong correlation between level of VEGF and ultrasound grading of RCD.

Key Words: rotator cuff, platelet rich plasma, cytokine.

\section{INTRODUCTION:}

Rotator cuff diseases (RCDs) are leading cause of shoulder pain and a significant source of disability and loss of work $^{(1,2)}$. Its prevalence increases substantial with age and with occupations involving overhead activities. They are affecting more than $50 \%$ of population above 60 years ${ }^{(3)}$.Disease course pass through 3 stages begin with acute tendinitis then progress to fibrosis and partial tear, end finally in full 
thickness tear ${ }^{(4)}$.Subacromial impingement, tendon degeneration, alternation in tendon mechanical properties, increase tendon overload and overuse especially with overhead activities are contributing factors in disease progression and development of partial and full thickness tears ${ }^{(3)}$.

Recent studies using immunehistochemistry techniques and synovial fluid samples revealed that the hallmark of RCD pathogenesis including proinflammatory, anti-inflammatory process, an abnormal immune response, angiogenesis and altered variables of vascularity ${ }^{(5)}$. Vascular endothelial growth factor (VEGF) found to be highly expressed in degenerated tendon. This cytokine thought to play a pivotal role in process of tendon degeneration and repair $^{(6)}$.As cytokines signal the normal process of inflammation and repair, they play important role in cell chemotaxis, proliferation, matrix synthesis and remolding. These molecules have the potential to improve RCT healing ${ }^{(6)}$. IL-1 $\beta$, TNF, IL-6, IL-10, proinflammatory cytokines are expressed in subacromial bursa in patients with $\operatorname{RCD}^{(7)}$. The combat between proinflammatory, antiinflammatory and angiogenic factors that end in a failed healing response, whichconsidered playing a principle part in pathogenesis of chronic tendon diseases ${ }^{(6)}$.

Management of RCD without full thickness tear is mainly conservativewith use of physiotherapy, manipulation, NSAID and local steroid injection but with high rate of recurrence and persistent pain ${ }^{(8,9)}$. Lack of healing response is the main cause of the unsatisfactory results of conservative treatment ${ }^{(10)}$. Therefore growth factors have been suggestive to be used to influence the healing process and promote tendon regeneration during treatment ${ }^{(11)}$.Platelets contain several cytokines and bioactive factors; PRP allows delivery of numerous cytokines in physiological balance ${ }^{(12)}$. The
PRP is expected to facilitate healing of poor vascular structure $^{(13)}$.

\section{AIM OF THE WORK:}

This study was aimed to assess the effect of PRP injection on functional improvement on RCD and to evaluate the role of proinflammatory and angiogenesisrelated cytokines as a pathogenic factor in RCD.

\section{PATIENTS AND METHODS:}

This study was conducted on 40 patients with RCD with age from 18-70 years and had un-satisfactory response to conservative therapy for at least 3 months. Criteria for diagnosis of RCD includes; shoulder pain with overhead activity more than 3 months, pain score more than 3 on visual analogue scale (VAS), painful arc or impingement signs, and were diagnosed with supraspinatus tendon disease on ultrasound examination $^{(14)}$.Informed consent was obtained. This study was approved by Research Ethics committee of the Faculty of Medicine, Ain Shams University. All patients were subjected to: clinical examination by history taking and full general and musculoskeletal examination with emphasis on shoulder examination, clinical scoring using (VAS and Los Angeles Shoulder score), cytokines assay of VEGF and IL-1 $\beta$ by ELISA assay using Human ELISA Kit, and diagnostic musculoskeletal ultrasound (MSU) examination and grading (grading of rotator cuff tendon:Grade 0: Normal (hyperechoic, fibrillaryechotexture); Grade 1: Mild tendinosis (heterogenousechotexture with ill-defined hyperechoicregions); Grade 2: Sever tendinosis (diffuse abnormal hypoechogenicitybut without tendon volume loss); Grade 3: Intrasubstance abnormality (focal, well-defined, hypoechoic area not extending to either the bursal or articular tendon surface); Grade 4: Partial-thickness 
tendon tear (focal, well-defined, hypoechoic area extending to either the bursal or articular tendon surface); Grade 5: Focal full-thickness tendon tear (focal, welldefined, hypoechoic area extending to either the bursal or articular tendon surface with tendon volume loss); Grade 6: Full-thickness tear (non-visualization of tendon with

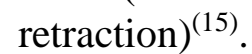

Forty Patients who met the inclusion criteria subjected to two doses of ultrasound guided PRP injection with 4 weeks between the first and second injection. A 3-week exercise program was done between the two doses followed by a further 3 -week exercise program after the second dose. PRP was prepared using a platelet concentration system using venipuncture for collection of $45 \mathrm{ml}$ of venous blood and then mixed with $5 \mathrm{ml}$ citrate, for the inhibition of clotting, a total of $50 \mathrm{ml}$ of blood will centrifuge in a special designed disposable tubes for double centrifugation; first for 15 minutes at 1600 rpm to separate the RBCs from plasma and second the plasma separated and centrifuged for 10 minutes at $3200 \mathrm{rpm}$ to separate platelet rich plasma from platelet poor plasma. Six ml of PRP was obtained; $1 \mathrm{ml}$ was collected for blood counting to establish the platelet concentration (at least double the serum plasma concentration) while the remaining $5 \mathrm{ml}$ of PRP without any buffering or activating agent was infiltrated under complete aseptic condition and under ultrasound guidance into the tendon partial tear.After injection, all patients were instructed to rest from overhead-throwing activity for 2 days. Acetaminophen and cold compression were allowed if needed; the use of NSAIDs was prohibited. After 2 days from injection, a 3-week exercise program will start under medical supervision. Reassessment after 4 weeks included; clinical scores, cytokines assay and MSU grading. Follow up assessment was done after 3 months and was included clinical scores and MSU grading.

Los Angeles Shoulder score is a combination of physical exam and subjective patient reported measures. It consists of 5 domains (pain, function, ROM, strength of forward flexion using manual muscle testing and patient satisfaction).A higher score indicates better function.

\section{Statistical analysis:}

Statistical presentation and analysis of our study was conducted and analyzed using the mean, standard deviation, repeated measure ANOVA, Chi-square test and paired sample t-test by SPSS program software version 17.

\section{RESULTS:}

The age of our patients ranged from 3464 years with a mean of $50.18 \pm 7.54$ years. Seventeen of patients were males $(42.5 \%)$ and 23 were females $(57.5 \%)$. Twenty-eight participants $(70 \%)$ had right shoulder affection and $12(30 \%)$ had left shoulder complaint. The duration of shoulder complaint in the patients was ranged from 124 months with a mean of $6.70 \pm 4.64$ months. The impingement tests werepositive in all participants before starting the injection protocol. The results of the rotator cuff integrity and other shoulder tests are shown in table (1). 
Table (1): Pre-injection Shoulder Clinical Special Tests.

\begin{tabular}{|ll|c|}
\hline \multicolumn{1}{|c|}{ Special Tests } & No. of positivity (40) and \% \\
\hline $1-$ & Impingement tests & 40 \\
\hline $2-$ & Rotator cuff strength and integrity tests: & $40(100 \%)$ \\
& Empty can test & $24(60 \%)$ \\
& External Rotation test & $20(50.0 \%)$ \\
lift-off test & $12(30.0 \%)$ \\
\hline $3-$ & Biceps tendon test & \\
\hline
\end{tabular}

Ultrasound shoulder grading of rotator cuff tendon was usedto assess the anatomical changes and the results were shown in table (2). Shoulder clinical scores were used to give quantitative data for comparison and their results were shown in table (3). Assessment of VEGF and IL-1 $\beta$ cytokines serum levels by ELISA were used to give quantitative data about the role of proinflammatory and angiogenesis-related cytokines in RCD, and their results were shown in table (3).

Table (2): Pre-Injection Musculoskeletal Ultrasound Assessment of Rotator Cuff.

\begin{tabular}{|l|c|}
\hline \multicolumn{1}{|c|}{ MSU } & No. (40) and \% \\
\hline Grade 0 and 1 & $0(0 \%)$ \\
\hline Grade 2 & $17(42.5 \%)$ \\
\hline Grade 3 & $13(32.5 \%)$ \\
\hline Grade 4 & $10(25.0 \%)$ \\
\hline Grade 5 and 6 & $0(0 \%)$ \\
\hline & \\
\hline
\end{tabular}

MSU: musculoskeletal ultrasound.

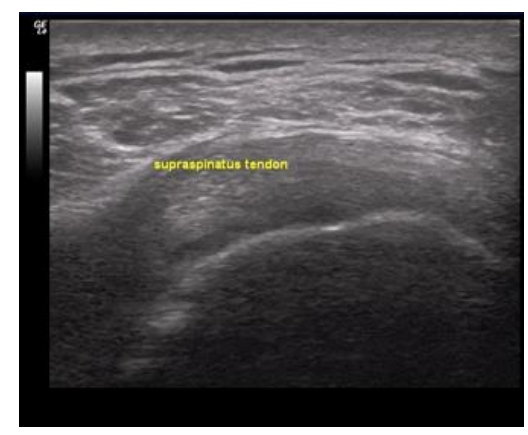

Figure 1: Grade 2 rotator cuff lesion (diffuse abnormal hypoechogenicity without tendon volume loss).

By the end of injection protocol, there was a highly statistical significant decrease in pain $(\mathrm{p}<0.01)$ assessed by VAS after 4 weeks and after 3 months, as shown in table (3). There was a highly statistical significant improvement of shoulder functionsin all participants after injection and rehabilitation program $(\mathrm{p}<0.01)$ after 4 weeks and after 3 months, table (3), assessed using Los Angeles Shoulder score. 
Table (3): Comparison between Different Clinical Scores before and after Injections.

\begin{tabular}{|c|c|c|c|c|c|c|}
\hline \multirow[t]{2}{*}{ Scores } & \multirow[t]{2}{*}{ Before Injection } & \multirow{2}{*}{$\begin{array}{c}\text { After } \\
4 \text { weeks }\end{array}$} & \multirow{2}{*}{$\begin{array}{c}\text { After } \\
3 \text { months }\end{array}$} & \multicolumn{3}{|c|}{ ANOVA test } \\
\hline & & & & $\begin{array}{c}\text { Test } \\
\text { value }\end{array}$ & $P$ & Sig. \\
\hline \multirow{2}{*}{$\begin{array}{l}\text { VAS } \\
\text { Range } \\
\text { Mean } \pm \text { SD }\end{array}$} & & & & \multirow[t]{2}{*}{333.978} & \multirow[t]{2}{*}{$<0.001$} & \multirow[t]{2}{*}{ HS } \\
\hline & $\begin{array}{c}4-9 \\
6.55 \pm 1.57\end{array}$ & $\begin{array}{c}2-6 \\
4.03 \pm 1.03\end{array}$ & $\begin{array}{c}0-5 \\
2.28 \pm 1.13\end{array}$ & & & \\
\hline \multirow{2}{*}{$\begin{array}{l}\text { Los Angeles Shoulder score } \\
\text { Range } \\
\text { Mean } \pm \text { SD }\end{array}$} & & & & \multirow[t]{2}{*}{618.183} & \multirow[t]{2}{*}{$<0.001$} & \multirow[t]{2}{*}{ HS } \\
\hline & $\begin{array}{c}8-24 \\
15.00 \pm 3.91\end{array}$ & $\begin{array}{c}16-28 \\
22.65 \pm 3.17\end{array}$ & $\begin{array}{c}24-34 \\
31.15 \pm 2.13\end{array}$ & & & \\
\hline
\end{tabular}

P-value >0.05: Non significant (NS); P-value <0.05: Significant (S); P-value< 0.01: highly significant (HS). VAS: visual analogue score; SD: standard deviation.

There was a highly statistical significant decrease of cytokines serum levels (VEGF and IL-1 $\beta$ ) after injection and rehabilitation program $(p<0.01)$ assessed by ELISA after 4

statistical significant increase in the number of patients that improved regarding MSU grading and become grade $1 \quad(p<0.01)$ assessed after 3 months, table (5). weeks, table (4). There was a highly Table (4): Comparison of Cytokines before and after Injection.

\begin{tabular}{|l|c|c|c|c|c|}
\hline Cytokines levels & Before Injection & After 4 weeks & \multicolumn{2}{|c|}{ Paired t- test } \\
\cline { 3 - 6 } & & & Test value & $P$ & Sig. \\
\hline VEGF (ng/L) & & & 11.367 & $<0.001$ & HS \\
Range & $800-3200$ & $400-1200$ & & & \\
Mean \pm SD & $1545.00 \pm 522.67$ & $737.50 \pm 209.62$ & & & \\
\hline IL-1 $\beta(\mathrm{pg} / \mathrm{L})$ & & & 10.125 & $<0.001$ & HS \\
Range & $1300-7800$ & $400-2400$ & & & \\
Mean \pm SD & $3587.50 \pm 1706.28$ & $1305.00 \pm 465.17$ & & & \\
\cline { 2 - 4 }
\end{tabular}

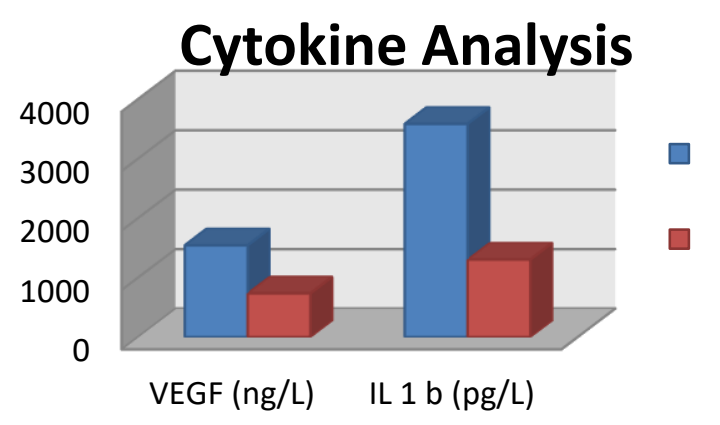

Figure 4: Comparison between cytokine analysis before and at week 4 after injection.

Table (5): Comparison of Musculoskeletal Ultrasound Assessment before and after Injection.

\begin{tabular}{|l|c|c|c|c|c|c|c|c|c|}
\hline \multirow{2}{*}{ MSU Grading } & \multicolumn{2}{|c|}{ Before } & \multicolumn{2}{c|}{ After 4 weeks } & \multicolumn{2}{c|}{ After 3 months } & \multicolumn{3}{c|}{ Chi-square test } \\
\cline { 2 - 11 } & \multicolumn{2}{|c|}{ No. 40} & \multicolumn{2}{c|}{ No. $=40$} & \multicolumn{2}{c|}{ No. $=40$} & Test value & $P$ & Sig. \\
\hline Grade 1 & 0 & $0.0 \%$ & 0 & $0.0 \%$ & 10 & $25.0 \%$ & 21.818 & $<0.001$ & HS \\
\hline Grade 2 & 17 & $42.5 \%$ & 18 & $45.0 \%$ & 13 & $32.5 \%$ & 1.458 & $>0.05$ & NS \\
\hline Grade 3 & 13 & $32.5 \%$ & 12 & $30.0 \%$ & 11 & $27.5 \%$ & 0.238 & $>0.05$ & NS \\
\hline Grade 4 & 10 & $25.0 \%$ & 10 & $25.0 \%$ & 6 & $15.0 \%$ & 1.571 & $>0.05$ & NS \\
\hline
\end{tabular}

P-value >0.05: Non significant (NS); P-value <0.05: Significant (S); P-value < 0.01: highly significant (HS). MSU: musculoskeletal Ultrasound.

There was a highly statistical significant positive correlation between the serum levels of cytokines (IL-1 $\beta$ and VEGF) and
VAS score $(\mathrm{p}<0.01)$ before injection and a statistically significant negative correlation between the serum levels of cytokines (IL- 
$1 \beta$ and VEGF) and Los Angeles Shoulderscore $(\mathrm{p}<0.05)$ before injection, as shown in table (6). There was a statistically significant positive correlation between the serum levels of VEGF and MSU grading of rotator cuff lesion $(\mathrm{p}<0.05)$ before injection, while no significant correlation between the serum levels of IL-1 $\beta$ and MSU grading of rotator cuff lesion $(\mathrm{p}>0.05)$.

Table (6): Correlation between Serum Levels of Cytokines and Other Methods of Assessments.

\begin{tabular}{|l|c|c|c|c|c|c|}
\hline \multirow{2}{*}{ Scores } & \multicolumn{3}{|c|}{ VEGF (ng/L) } & \multicolumn{3}{c|}{ IL-1 $\beta(\mathrm{pg} / \mathrm{L})$} \\
\cline { 2 - 7 } & $\mathrm{r}$ & $p$ & Sig. & $\mathrm{r}$ & $P$ & Sig. \\
\hline VAS & 0.523 & $<0.001$ & HS & 0.872 & $<0.001$ & HS \\
\hline Los Angeles Shoulder score & -0.437 & $<0.001$ & HS & -0.713 & $<0.001$ & HS \\
\hline MSU Grading & 0.384 & $<0.05$ & S & 0.113 & $>0.05$ & NS \\
\hline
\end{tabular}

P-value >0.05: Non significant (NS); P-value <0.05: highly significant (HS).

\section{DISCUSSION:}

The protocol we used in our study has shown successful results, with no significant side effects, in form of a high statistically significant decrease in pain assessed by VAS as well as a high statistically significant improvement of shoulder in all participants assessed using Los Angeles Shoulder score. This clinical improvement results following PRP therapy was similar to results of other researchers $(16,17,18)$. The main targets of PRP therapy are to curb the inflammatory response and supplement tendon-bone healing with growth factors ${ }^{(13)}$. In addition, PRP stimulates cell proliferation and synthesis of extracellular matrix ${ }^{(4,20)}$. All of these benefits are added to the fact that no reported negative outcomes from PRP use has been published till now ${ }^{(21,22)}$, so it could consider as a good option in patient for whom steroid use is a concern.In contrast to our study, Rha and his collogue in 2013reported no clear benefit of PRP therapy over other conservative measures ${ }^{(23)}$.In their study, they reported that the disease-specific scores for the PRP group improved at 1-year follow-up but this improvement did not differ from the placebo group $^{(23)}$.This can be explained as the patients received single injection of PRP therapy, improvement in placebo group can be explained by the needling effect. The needling causes focal bleeding, so it can stimulate an inflammatory response and promote healing in tendon tissue ${ }^{(24)}$.

Despite the clinical improvement after 4 weeks, yet there was a continued clinical improvement with a high statistically significance after second injection. Serial injection is one of the controversial topics in PRP application. In our study we use second injection 4 weeks after the first one to maximize the healing effect of PRP therapy especially that the healing rate of rotator cuff tendons are slow. In accordance, some researchers suggested that there was an improved result obtained during the 3-month follow-up and it could be related to a delayed effect of the first PRP injection or to a boosting effect of the second injection ${ }^{(17)}$. Furthermore, objective sonographic tendon improvements cannot be visualized at the time of initial follow-up and are generally not used to determine the need for additional injections. Complete tendon remodeling take up to 2 years to occur, leaving the clinician with few objective data to guide the decision to repeat.

One purpose of this study was to evaluate the role of pro-inflammatory cytokines (as IL-1 $\beta$ ) and vascular angiogenesis-related cytokines (as VEGF) as a pathogenic factor in RCD. In normal individual, IL-1 $\beta$ is under the lower limit of detection (LLOD), either because levels were very low or because these molecules are not produced by healthy subjects ${ }^{(25)}$. On 
other hand, the normal value of VEGF is expected to be less than 500ng/L (26). Regarding our study, the baseline concentration of serum levels of IL- $1 \beta$ and VEGF were high. The pathogenesis of RCD is associated with an imbalance between different cytokines. The changes of these cytokines create an imbalance between degeneration and repair. In addition, degenerative changes that occur in RCD enhance production of pro-inflammatory cytokines and the vascular angiogenesisrelated cytokines as they play a pivotal role in the pathogenesis of RCD and to improve the healing process ${ }^{(6)}$. In accordance, increased serum levels of IL-1 $\beta$ in RCD patients are a diagnostic indicator of the inflammatory phase.

Four weeks following injection, there was high statistically significant decrease of cytokines levels. This decrease went hand by hand with the clinical improvement. The initial high levels of these cytokines and the subsequent significant decrease with clinical improvement suggest the role of these cytokines in the pathogenesis of RCD as well as the effectiveness of our protocol in management of RCD. Three months following the first injection showed radiological improvement although it did not show statistically significant changes. This reflects on going healing of the injured ligaments as well as the effectiveness of injection and rehabilitation program. Despite the clinical improvement after 4 weeks, yet there was no improvement in radiological grading of rotator cuff lesion 4 weeks after first injection. This was similar to the finding of many other studies who reported that MRI and ultrasound findings after treatment hardly changed although clinical improvement ${ }^{(17)}$. This highlights that functional recovery precedes the anatomical recovery which is assessed using ultrasound $^{(27)}$.Among our study, there was a high statistically significant positive correlation between the baseline serum levels of cytokines and VAS score before injection and a statistically significant negative correlation between the serum levels of these cytokines and Los Anglos score before injection. These correlations prove the role of these cytokine in pathogenesis of RCD; as with their higher levels, there was worse clinical state.

\section{Conclusion}

PRP therapy is an effective treatment for RCT and lead to functional improvement. There is a strong correlation between levels of cytokines (IL-1 $\beta$ and VEGF) and clinical scores before and after injection. There is strong correlation between level of VEGF and ultrasound grading of rotator cuff lesion.

\section{REFERENCES:}

1. Rha D, Park G, Kim Y, Kim MT and Lee SC (2012): Comparison of the Therapeutic Effects of Ultrasound-Guided Platelet-Rich Plasma Injection and Dry Needling in Rotator Cuff Disease: A Randomized Controlled Trial. Clinical Rehabilitation, 27(2) 113-22.

2. Whittle S and Buchbinder R (2015):In the clinic. Rotator cuff disease. Ann Intern Med, 162: Itc1-15.

3. Seitz AL, McClure PW, Finucane S, Boardman ND 3rd and Michener LA (2011): Mechanisms of Rotator Cuff Tendinopathy: Intrinsic, Extrinsic or Both? ClinBiomech., 26:1-12.

4. Kesikburun S, Tan AK, Yilmaz B, Yas, ar E and Yaziciog lu K (2013): Platelet-rich plasma injections in the treatment of chronic rotator cuff tendinopathy a randomized controlled trial with 1-year follow-up. Am J Sports Med, 41.

5. Löhr JF and Uhthoff HK (2007): Epidemiology and Pathophysiology of Rotator Cuff Tears. Orthopade, 36(9):78895.

6. Savitskaya YA, Izaguirre A, Sierra L, Perez F, Cruz F, Villalobos E, Almazan A and Ibarra C (2011): Effect of Angiogenesis- 
Related Cytokines on Rotator Cuff Disease: the Search for Sensitive Biomarkers of Early Tendon Degeneration. Clinical Medicine Insights: Arthritis and Musculoskeletal Disorders, 4: 43-53.

7. Voloshin I, Gelinas J, Maloney MD, O'Keefe RJ, Bigliani LU and Blaine TA (2005): Proinflammatory Cytokines and Metalloproteases Are Expressed in the Subacromial Bursa in Patients with Rotator Cuff Disease. The Journal of Arthroscopic and Related Surgery, 21(9): 1076.e11076.e9.

8. Andrews JR (2005): Diagnosis and Treatment of Chronic Painful Shoulder: Review of Nonsurgical Interventions. Arthroscopy, 21: 333-47.

9. Chou WY, Ko JY, Wang FS, et al. (2010): Effect of Sodium Hyal huronate Treatment on Rotator Cuff Lesions Without Complete Tears: A Randomized, Double-Blind, Placebo-Controlled Study. J Shoulder Elbow Surg, 19: 557-63.

10. Rees JD, Wilson AM and Wolman RL (2006): Current Concepts in the Management of Tendon Disorders. Rheumatology (Oxford), 45:508-21.

11. Wu G, Xu PC, Wu P, Hu K, Sun Y, Cheng $\mathrm{B}$ and $\mathrm{Lu} \mathrm{Y}$ (2017): Advances in the treatment of rotator cuff lesions by cytokines. Frontiers in Bioscience, 22.

12. Rodeo SA, Delos D, Williams RJ, Adler RS, Pearle A and Warren RF (2012): The effect of platelet-rich fibrin matrix on rotator cuff tendon healing a prospective, randomized clinical study. Am J Sports Med, 40: 1234-41.

13. Pauyo T and Bradley JP (2018): Use of PRP in Rotator Cuff Repair. In McMahon PJ. Rotator Cuff Injuries: A Clinical Casebook. Springer International Publishing AG, 18: 277-92.

14. Roy A, Kishner S, AdahanThHM, Bélair M and Dahan B (2016): Rotator Cuff Disease. Medscape.

15. Collinger JL, Fullerton B, Impink BG, Koontz AM and Boninger ML (2010): Validation of Greyscale-Based Quantitative
Ultrasound in manual Wheelchair Users: Relationship to Established Clinical Measures of Shoulder Pathology. Am J Phys Med Rehabil, 89(5): 390- 400.

16. Balasubramaniam U, Dissanayake $\mathrm{R}$ and Annabell L (2015): Efficacy of platelet rich plasma injections in pain associated with chronic tendinopathy: a systematic review. PhysSportsmed, 43: 253-61.

17. Nejati $P$, Ghahremaninia A, Naderi F, Gharibzadeh $\mathrm{S}$ and Mazaherinezhad A (2017): Treatment of subacromial impingement syndrome: platelet-rich plasma or exercise therapy? A randomized controlled trial. Orthop J Sports Med, 5(5).

18. Ibrahim DH, El-Gazzar NM, El-Saadany HM and El-Khouly RM (2019): Ultrasound-guided injection of platelet rich plasma versus corticosteroid for treatment of rotator cuff tendinopathy: Effect on shoulder pain, disability, range of motion and ultrasonographic findings. The Egyptian Rheumatologist, 41: 157-61.

19. deVos RJ, Weir A, van Schie HT, BiermaZeinstra SM, Verhaar JA, Weinans $\mathrm{H}$ and Tol JL (2010): Platelet rich plasma injection for chronic Achilles tendinopathy: a randomized controlled trial. JAMA, 303(2): 144-9.

20. Hoppe S, Alini M et al., (2012): Tenocytes of chronic rotator cuff tendon tears can be stimulated by platelet-released growth factors. J Shoulder Elbow Surg.

21. Kaux JF, Croisier JL, Léonard P, Le Goff C and Crielaard JM (2014): Exuberant inflammatory reaction as a side effect of platelet-rich plasma injection in treating one case of tendinopathy. Clin J Sport Med, 24: $150-2$

22. Kaux JF (2019): Platelet-rich plasma for treating chronic tendinopathy. Aspetar Sport Medicine Journal, 118-22.

23. Rha D, Park GY, Kim YK, Kim MT and Lee SCh (2013): Comparison of the therapeutic effects of ultrasound-guided platelet-rich plasma injection and dry needling in rotator cuff disease: a randomized controlled trial. ClinRehabil, 27(2). 
24. Nguyen RT, Borg-Stein $\mathrm{J}$ and McInnis $\mathrm{K}$ (2011): Applications of Platelet-Rich Plasma in Musculoskeletal and Sports Medicine: An Evidence-Based Approach. PM R, 3:226-50.

25. Kleiner G, Marcuzzi A, Zanin V, Monasta L and Zauli G (2013): Cytokine Levels in the Serum of Healthy Subjects. Mediators of Inflammation.

26. Larsson A, Sköldenberg E and Ericson $\mathrm{H}$ (2002): Serum and plasma levels of FGF-2 and VEGF in healthy blood donors. Angiogenesis, 5(1-2): 107-10.
27. Dallaudie're B, Meyer $\mathrm{P}$, Hummel $\mathrm{V}$, Perozziello A, et. al., (2013): efficacy of second intra-tendinous platelet-rich-plasma injection in case of incomplete response of the first injection: Three-year follow up experience. Diagnostic and Interventional Imaging, 94: 871-7.

28. CroyTh, Saliba S, Saliba E, Anderson MW and Hertel J (2013): Talofibular Interval Changes After Acute Ankle Sprain: A Stress Ultrasonography Study of Ankle Laxity. Journal of Sport Rehabilitation, 22: 257-63. 


\author{
دور العلاج بالبلازما الغتيه بالصفائح الامويه في متلازمة الكم الدوار

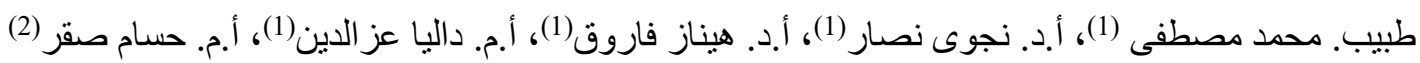

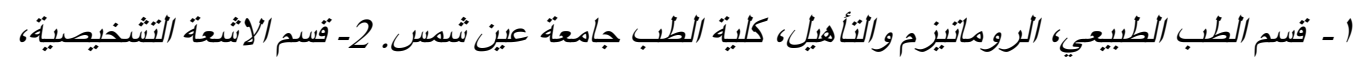 \\ كلية الطب جامعة عبن شعس النس
}

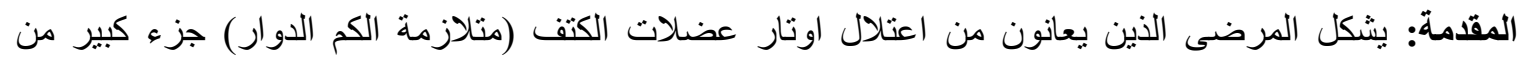

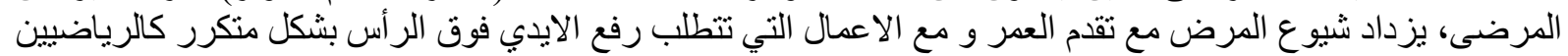

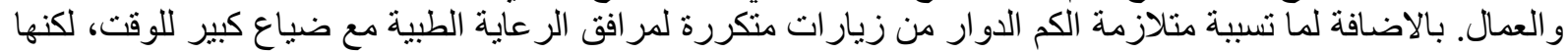
ايضا قد تسبب العجز على المدى الطويل.

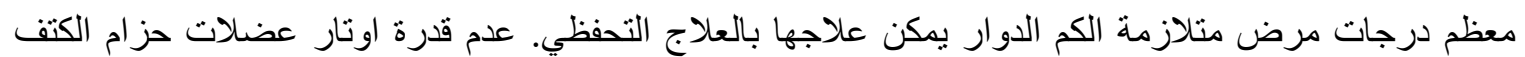

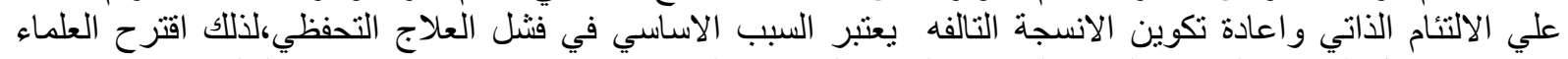
استخدام عو امل النمو او البلازما الغنية بالصفائح الدموية لتحفيز الالتنئام وسرعة الإنادية التادية تكوين الانسجة التالفه.

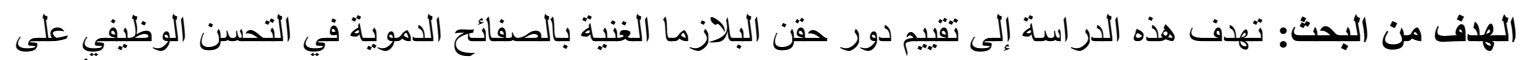

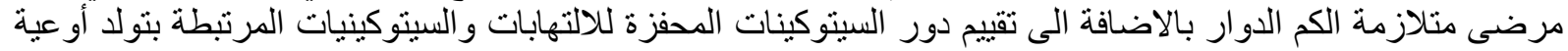
جديدة كعامل ممرض في مرضى متلازمة الكم الدوار

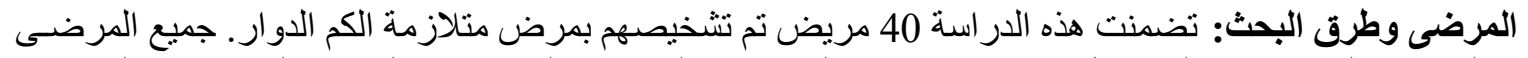

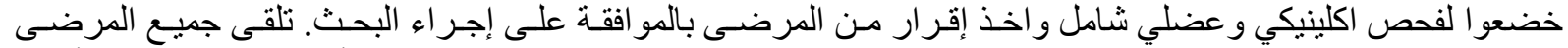

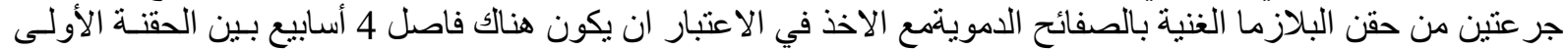

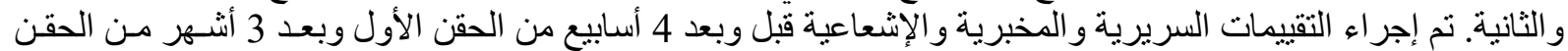

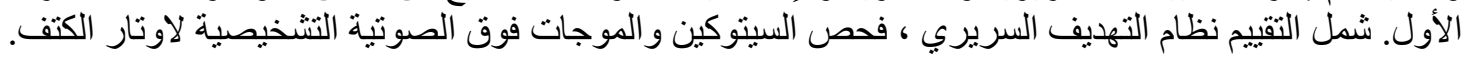

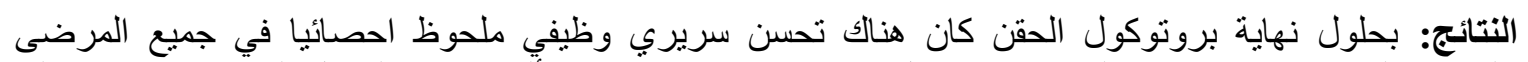

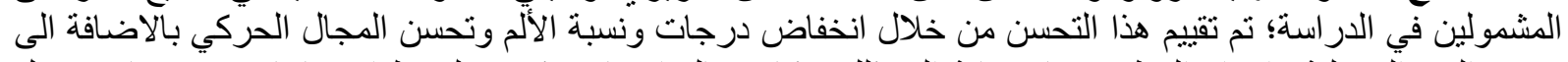

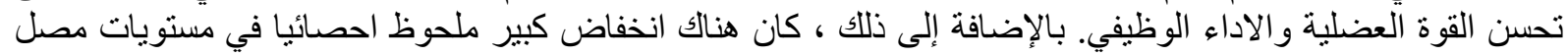

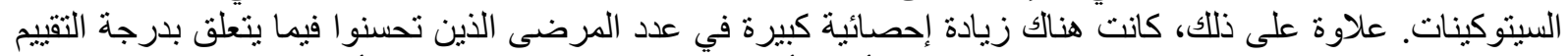

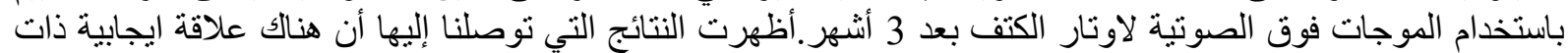

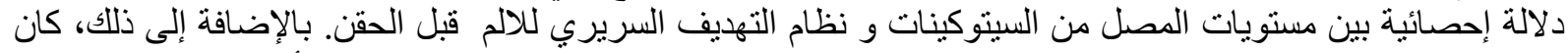

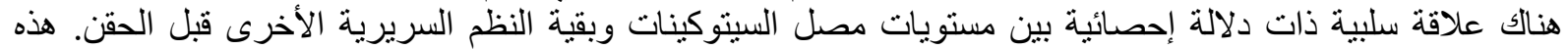
الارتباطات تثبت دور السيتوكين في التسبب في مرض دلة متلازمة الكم الدوار. 